\section{$02-6.3$ \\ THE RISK FOR AUTISM AND FOR AUTISM WITH CO- EXISTING DEVELOPMENTAL DISABILITIES IN LOW BIRTH WEIGHT CHILDREN COMPARED TO NORMAL BIRTH WEIGHT CHILDREN}

\author{
doi:10.1136/jech.2011.142976a.78
}

${ }^{1} \mathrm{~L}$ Hjort, ${ }^{*}{ }^{2} \mathrm{M}$ B Lauritsen, ${ }^{3}$ E Parner. ${ }^{1}$ Department of Epidemiology, School of Public Health, Aarhus University, Aarhus, Denmark; ${ }^{2}$ Aarhus University Hospital, Regional Center for Child and Adolescent Psychiatry, Risskov, Aarhus, Denmark; ${ }^{3}$ Department of Biostatistics, School of Public Health, University of Aarhus, Aarhus, Denmark

Introduction Recently, the autism age-specific prevalence has increased dramatically and autism prevalence estimates now range from 60 to 70/10 000. This makes autism the second most common neurodevelopmental disorder after mental retardation (MR) and a serious public health concern.

Autism is a highly heritable multifactorial disorder, but the specific etiological pathways are largely unknown. Previous studies reported ORs from 1.9 to 2.3 for autism in low birth weight children (lbw, <2500 g) when compared to normal birth weight children (nbw, 3000-3999 g), but most studies have ignored the possible impact of comorbidities. Co-existing developmental disabilities (CDDs) like $\mathrm{MR}, \mathrm{ADHD}$, and epilepsy are common in autism.

We hypothesise that lbw is a stronger risk factor for autism with CDDs than for autism without CDDs. We report HR (95\% CI) for autism + CDDs and autism / noCDDs specifically in lbw children when compared to nbw children.

Methods We conducted a nationwide, register-based, follow-up study. 1990 to 2007 birth cohorts were identified in The Danish Medical Birth Register and followed through 2009. Linkage with national hospital registers made exposure, outcome, and covariates information available to us.

Results We found HR 2.0 (1.5 to 2.8) for autism + MR and HR 0.9 (0.7 to 1.1) for autism/no MR in lbw children when compared to nbw children. Analyses of more CDD subgroups are ongoing.

Conclusion Consistent with a recently published study we find that lbw children have a twofold increased risk for autism with MR but no increased risk for autism with normal intelligence. In etiological studies, subgrouping autism cases on the basis of CDDs may enhance our knowledge of etiological pathways in autism.

\section{2-6.4 IMPACT OF MATERNAL OBESITY ON STILLBIRTH AND INFANT DEATH: ABSOLUTE RISK AND TEMPORAL TRENDS}

doi:10.1136/jech.2011.142976a.79

${ }^{1}$ P W G Tennant, ${ }^{* 1,2} \mathrm{~J}$ Rankin, ${ }^{1,2} \mathrm{R}$ Bell. ${ }^{1}$ Institute of Health and Society, Newcastle University, Newcastle upon Tyne, UK; ${ }^{2}$ Regional Maternity Survey Office, Newcastle upon Tyne, UK

Introduction UK guidelines advocate that obese pregnant women (body mass index, BMI $\geq 30 \mathrm{~kg} / \mathrm{m}^{2}$ ) be made aware of the increased risks to them and their offspring. This study hence pooled data from several sources to derive estimates of the absolute and attributable risks of stillbirth and infant death for obese women in England, and predict changes in prevalence resulting from trends in BMI

Methods The BMI profile of the maternal population of England and of the prevalence of each outcome were obtained from nationally representative sources. Trends in BMI were modelled by logistic regression. RRs for stillbirth and infant death were derived from published literature. These were equated to estimate absolute risks, attributable risks, and future prevalence rates.

Results The estimated absolute risk of a stillbirth or infant death for an obese pregnant woman in England is 1.5\% (95\% CI 1.3 to 1.8), compared to $0.9 \%$ (0.8 to 0.9 ) for women of recommended BMI $\left(25-29 \mathrm{~kg} / \mathrm{m}^{2}\right)$. An estimated $8.1 \%$ of stillbirths and infant deaths in England are attributable to maternal obesity.

If trends in maternal BMI continue, $24.0 \%$ (22.1 to 25.9) of the maternal population of England will be obese by 2020. This is predicted to result in a $4.4 \%$ increase in the prevalence of stillbirth and infant death compared to 2010 .

Conclusion This study provides estimates of the individual risk and population burden of stillbirth and infant death in England resulting from maternal obesity. These results have implications for public health planning and for providing clear information to obese women about their pregnancy-related risks

\section{2-6.5 RISK OF FETAL DEATH IN WOMEN WITH PERICONCEPTIONAL INTAKE OF MULTIVITAMINS}

doi:10.1136/jech.2011.142976a.80

${ }^{1}$ E A Nohr, ${ }^{1}{ }^{1} \mathrm{~J}$ Olsen, ${ }^{1} \mathrm{~B}$ H Bech, ${ }^{2} \mathrm{~L}$ Bodnar, ${ }^{3} \mathrm{~J} \mathrm{M}$ Catov. ${ }^{1}$ Institute of Public Health, Department of Epidemiology, Aarhus University, Aarhus, Denmark; ${ }^{2}$ Department of Epidemiology, University of Pittsburgh Graduate School of Public Health, Pittsburgh, Pennsylvania, USA; ${ }^{3}$ Department of Obstetrics, Gynecology \& Reproductive Sciences, University of Pittsburgh, Pittsburgh, Pennsylvania, USA

Introduction Nutrition is important in a healthy pregnancy, but little is known about the impact of multivitamins (MV) on the survival of the fetus.

Methods We related periconceptional MV use to early ( $<20$ weeks) and late ( $\geq 20$ weeks) fetal death. At recruitment, women in the Danish National Birth Cohort $(n=35897)$ reported the number of weeks of MV use during a 12 week periconceptional period. Information about lifestyle factors came from a later telephone interview. Cox regression was used to estimate HR for the association between MV use and fetal death with follow-up starting at 8 completed weeks of gestation. Intensity of preconception use ( 6 weeks before conception) and postconception use ( 6 weeks after conception) were categorised as use in $1-2,3-4$, and $5-6$ weeks. No use at any time of these weeks was reference.

Results Compared to women with $<2$ weeks of preconception $\mathrm{MV}$ use, risk of early fetal death increased with increasing intensity of 
use (adj. HRs 1.18 [0.84 to 1.67] and 1.31 [1.03 to 1.66] for 3-4 and 5-6 weeks of use, respectively). Risk of early fetal death was also increased in women with the highest intensity of post-conception use (adj. HR 1.84 [1.11 to 3.03] while for late fetal death, we identified a reduced risk with high intensity exposure (adj. HR 0.56 [0.32 to 0.97] for 5-6 weeks of use).

Conclusion While these results indicate that postconception use of MV has a preventive effect on late fetal death, MV use was consistently associated with a higher rate of early fetal death.

\section{2-6.6 MATERNAL SMOKING DURING PREGNANCY AND SMOKING IN THE OFFSPRING WHO WERE FOLLOWED FROM BIRTH TO ADULTHOOD: FINDINGS FROM THE 1958 NCDS BRITISH BIRTH COHORT}

doi:10.1136/jech.2011.142976a.81

${ }^{1,2}$ D Canoy. ${ }^{1}$ Cancer Epidemiology Unit, University of Oxford, Oxford, UK; ${ }^{2}$ School of Community-Based Medicine, The University of Manchester, Manchester, UK

Background Early life factors, such as smoking during pregnancy, have been suggested to influence smoking in the offspring particularly during teenage years. It remains unclear if this relation persists over time considering that longer duration and increased amounts of smoking are associated with premature deaths.

Method The prospective relation of maternal smoking during pregnancy and smoking in 15151 offspring in a British birth cohort (1958 National Child Development Study) over the life course was investigated using logistic regression.

Results Heavy smoking ( $\geq 10$ cigarettes/day) during (but not before) pregnancy was related to increased risk of heavy smoking in the offspring, with sex- and concurrent social class-adjusted OR [95\% CI] of 1.32 (1.16 to 1.50 ), 1.60 (1.39 to 1.84 ), 1.82 (1.56 to 2.13 ), 1.46 (1.21 to 1.76$), 1.53$ (1.26 to 1.84$)$ and 1.51 (1.17 to 1.94$)$ at ages 16 , $23,42,46$ and 50 years, respectively. The OR for being a heavy smoker across all ages was 5.25 (95\% CI 2.65 to 10.40), after adjusting for sex and social class across the life course.

Conclusion The consistency and magnitude of the risk estimates suggest that the association could be aetiologically important but the absolute risk due to maternal smoking during pregnancy was small, suggesting limited public health relevance. Other factors, such as social circumstances, are more important considerations for addressing the burden of smoking in the population. 